\title{
The Direct Contribution of Astrocytes and Microglia to the Pathogenesis of Hepatic Encephalopathy
}

\author{
Victoria Jaeger ${ }^{1}$, Sharon DeMorrow ${ }^{2,3,4,5}$ and Matthew McMillin*2,3,4 \\ ${ }^{1}$ Baylor Scott \& White Health, Department of Internal Medicine, Temple, TX, USA; ${ }^{2}$ Texas A\&M University Health Science Center, \\ Department of Medical Physiology, Temple, TX, USA; ${ }^{3}$ Central Texas Veterans Health Care System, Temple, TX, USA; ${ }^{4}$ University \\ of Texas at Austin, Dell Medical School, Department of Internal Medicine, Austin, TX, USA; ${ }^{5}$ University of Texas at Austin, College \\ of Pharmacy, Austin, TX, USA
}

\begin{abstract}
Hepatic encephalopathy is a neurological complication resulting from loss of hepatic function and is associated with poor clinical outcomes. During acute liver failure over $20 \%$ of mortality can be associated with the development of hepatic encephalopathy. In patients with liver cirrhosis, 1-year survival for those that develop overt hepatic encephalopathy is under $50 \%$. The pathogenesis of hepatic encephalopathy is complicated due to the multiple disruptions in homeostasis that occur following a reduction in liver function. Of these, elevations of ammonia and neuroinflammation have been shown to play a significant contributing role to the development of hepatic encephalopathy. Disruption of the urea cycle following liver dysfunction leads to elevations of circulating ammonia, which enter the brain and disrupt the functioning of astrocytes. This results in dysregulation of metabolic pathways in astrocytes, oxidative stress and cerebral edema. Besides ammonia, circulating chemokines and cytokines are increased following liver injury, leading to activation of microglia and a subsequent neuroinflammatory response. The combination of astrocyte dysfunction and microglia activation are significant contributing factors to the pathogenesis of hepatic encephalopathy.
\end{abstract}

Citation of this article: Jaeger V, DeMorrow S, McMillin M. The direct contribution of astrocytes and microglia to the pathogenesis of hepatic encephalopathy. J Clin Transl Hepatol 2019;7(4):352-361. doi: 10.14218/JCTH.2019.00025.

\section{Introduction}

Liver disease is a significant cause of morbidity and mortality worldwide. One of the most severe consequences of acute liver failure and decompensated chronic liver disease is the development of neurological complications, a state referred

Keywords: Acute liver failure; Microglia; Neuroinflammation; Ammonia; Astroglia.

Abbreviations: $A Q P-4$, aquaporin-4; $B D L$, bile duct-ligated; $C C L 2$, chemokine ligand 2; CNS, central nervous system; CX3CL1, C-X3-C ligand 1; GFAP, glial fibrillary acidic protein; $\mathrm{HE}$, hepatic encephalopathy; IL, interleukin; IBA1, ionized calcium binding adaptor molecule 1 ; LPS, lipopolysaccharide; TGF $\beta 1$, transforming growth factor beta 1 ; TGF $\beta R 2$, transforming growth factor beta receptor 2; TNF $\alpha$, tumor necrosis factor alpha; WHC, West Haven Criteria.

Received: 5 July 2019; Revised: 7 October 2019; Accepted: 24 October 2019

* Correspondence to: Matthew McMillin, University of Texas at Austin Dell Medical School, 1601 Trinity Street, Building B, Austin, TX 78701, USA. Tel: +1-512-4955037, Fax: +1-512-495-5839, E-mail: matthew.mcmillin@austin.utexas.edu to as hepatic encephalopathy (HE). HE manifests in several different ways and while the severity of symptoms is variable, patients often present with altered mental status, disturbances in sleep-wake cycles and functional impairment. $^{1,2}$ Hospitalizations due to HE in the USA increased over the period of 2010 to 2014 , from 25,039 to 31,182 , though in-hospital mortality decreased from $13.4 \%$ to $12.3 \% .^{3}$ In addition to this, HE in-patient charges increased from 8.15 billion USD to 11.9 billion USD over the same time period. ${ }^{3}$ Therefore, it is evident that HE is a significant financial burden on health care systems and the development of this syndrome is associated with poor patient prognosis and mortality.

The current understanding of HE pathogenesis is that disruption of hepatic function leads to increased ammonia concentrations and impaired urea metabolism, which allows ammonia to accumulate in the blood and the brain. The downstream effects of ammonia accumulation in the brain are astrocyte swelling and dysregulated neurotransmission, which lead to the cognitive deficits present during HE. In addition to this, systemic inflammation, elevation of proinflammatory cytokines and microglia activation have been observed during $\mathrm{HE}$ in both patients and rodent models and are associated with worse outcomes. Current treatments are primarily focused on ammonia-lowering agents, optimization of nutrition and treatment of comorbid disease processes. While these have been shown to have some efficacy, determining all of the pathophysiological mechanisms that contribute to HE progression is important if novel therapeutic approaches are to be developed.

In this review, we will discuss the clinical definitions and description of $\mathrm{HE}$, further examine the available research regarding the pathophysiology of $\mathrm{HE}$ within the central nervous system (CNS), and more specifically discuss the role of astrocytes and microglia within the context of $\mathrm{HE}$ pathology.

\section{Clinical epidemiology and description of HE}

The neurological and cognitive manifestations that present in patients with liver disease or portosystemic shunting in the absence of liver disease is termed HE. ${ }^{4}$ Two categories of $\mathrm{HE}$ exist: covert $\mathrm{HE}$ (also known as minimal $\mathrm{HE}$ ) and overt $\mathrm{HE}$; these are distinguished by the severity of the patient presentation. ${ }^{5}$ Patients with minimal HE have abnormal results with psychometric testing and subtle personality changes that may be evident to caregivers or by those familiar with the 
patient. Individuals with minimal $\mathrm{HE}$ can have a reduced ability to drive, impairment at work, and inability or difficulty performing other complex tasks. ${ }^{6-8}$ Diagnosing minimal HE is important clinically because it best predicts future diagnosis of overt HE and is associated with higher rates of hospitalization and lower survival rates. ${ }^{6,9}$ Overt HE is more evident upon clinical presentation, with increased severity of symptoms, but is diagnosed only after exclusion of other causes of altered mental status.

The West Haven Criteria (WHC) have traditionally been used for grading the severity of overt HE based upon clinical findings, with higher WHC grades being associated with more severe HE. The mortality rate is high for overt HE patients regardless of grade. During acute liver failure, $20-25 \%$ of mortality results from increased intracranial pressure and the development of HE. ${ }^{10}$ In cirrhotic patients, survival rates are estimated to be $42 \%$ at 1 year and $23 \%$ at 3 years. ${ }^{11}$ The incidence of overt HE is $8 \%$ annually in decompensated cirrhotic patients ${ }^{12}$ and is estimated to be attributed to $0.33 \%$ of hospitalizations in the USA. ${ }^{9}$

The American Association for the Study of Liver Diseases (commonly known as AASLD) together with the European Association for the Study of the Liver (commonly known as EASL) established guidelines in 2014 consisting of four axes for better categorization and management of $\mathrm{HE}$ in the setting of chronic liver disease. ${ }^{6}$ These guidelines are focused on etiology, severity, time course, and inciting factors. Axis I is categorized via etiology as Type A (acute liver failure), Type $B$ (portosystemic shunting), or Type C (cirrhosis). Axis II is determined by clinical severity of symptoms and is categorized as unimpaired (normal mentation), WHC Grade I (abnormal psychometric testing without apparent mental status changes), WHC Grade II (evidence of disorientation or asterixis), WHC Grade III (gross disorientation, somnolence), and WHC Grade IV (coma). ${ }^{4,6}$ Axis III is based on time of the clinical course and is categorized as episodic (more than 6 months between episodes), recurrent (episodes occurring within 6 months), or persistent (always present). ${ }^{6,9}$ Axis IV is categorized as spontaneous or precipitated. Documentation of a patient with HE should include all four axis components and can aid clinicians in consistent categorization of this patient population.

\section{Pathogenesis of HE}

The pathogenesis of HE is complex and the exact disease mechanisms have yet to be fully delineated. The most common theory is that the manifestations of HE are due to alteration of the urea cycle and the resulting increase of systemic ammonia levels. ${ }^{4}$ Glutamine is used by the gut as a primary energy source and the resulting by-products of its metabolism by glutaminase are glutamate and ammonia. In addition, bacteria in the gastrointestinal tract use urase to produce ammonia from urea. Ammonia from the gut diffuses through the intestinal mucosa, enters the mesenteric circulation and is transported to the liver. In the liver, periportal hepatocytes detoxify ammonia via the ornithine cycle, resulting in the production of urea. Urea passes into the systemic circulation via the hepatic veins, where it enters the kidney and is ultimately excreted in urine. Ammonia that does not enter the urea cycle is metabolized by glutamine synthetase in the liver, kidney, muscle and brain to produce glutamine from glutamate. ${ }^{13}$
When hepatocytes are damaged as a result of liver disease, detoxification of ammonia into urea is impaired and portosystemic shunting leads to ammonia accumulation in the blood. In addition, dysbiosis of the microbiome has been reported during cirrhosis, leading to increased urase activity and even greater production of ammonia in the gut. ${ }^{14}$ These combined effects lead to a significant increase of circulating ammonia concentrations, which are then metabolized by muscle and other extrahepatic organs. Ammonia also can cross the blood-brain barrier, generating a state of hyperammonemia in the brain. It is not clear whether the increased concentrations of ammonia found in the brain and cerebrospinal fluid are due to active or passive transport but increased systemic levels of ammonia are associated with increased ammonia concentrations in the brain. ${ }^{15}$ The highest elevations of serum ammonia following liver dysfunction are observed in Type A and Type B HE. ${ }^{16,17}$ Arterial and venous ammonia have been reported to be elevated during Type $\mathrm{CHE}$ compared to controls, though the values observed are lower than the other types of $\mathrm{HE}$, including in patients with acuteon-chronic liver failure. ${ }^{16}$

Besides ammonia, acute liver injury or chronic liver disease is associated with inflammation that contributes to this syndrome, with neuroinflammation being a pathological contributor to overt $\mathrm{HE}$ during both acute liver failure and chronic liver disease. Elevation of circulating pro-inflammatory cytokines has been observed during acute liver failure, cirrhosis, and acute-on-chronic liver failure. ${ }^{18-20}$ Associated with increased systemic inflammation, is the development of neuroinflammation and $\mathrm{HE}$. In the brain, neuroinflammation is regulated by microglia, the resident macrophage-like cells of the brain. While normally present in a quiescent state, in response to pro-inflammatory signals, they can become activated, producing cytokines generating oxidative stress and contributing to aspects of pathology, and activation of microglia is observed in patients with both Type A and Type C HE. ${ }^{21}$

In addition to increased ammonia and inflammation are a variety of other dysregulated metabolites that can contribute to this syndrome. The concentrations of various amino acids have been shown to be outside the normal range when assessed in plasma, cerebrospinal fluid and frontal cortex of patients with Grade 3 or $4 \mathrm{HE} .{ }^{22}$ Similar findings have been observed for bile acids, where concentrations are increased in serum, cerebrospinal fluid and tissue from patients with fulminant hepatic failure. ${ }^{23}$ Manganese is normally excreted from the body through the hepatobiliary system but during cirrhosis has been shown to accumulate in both the circulation and brain, and can inhibit dopaminergic neurotransmission in the basal ganglia. ${ }^{24-26}$

In addition to this, a variety of comorbidities, including infections, gastrointestinal bleeding, diarrhea, hyponatremia, hypokalemia, hyperkalemia, benzodiazepine and diuretic use, and others contribute to this syndrome. ${ }^{27}$ These comorbidities are most often present in patients with liver cirrhosis. In acute-on-chronic liver failure patients, elevated creatinine, as well as altered jugular venous oxygen saturation ranges, were associated with higher grades of HE. ${ }^{28}$ This gives support that multiorgan complications during cirrhosis and acute-on-chronic liver failure can exacerbate the development of HE. A diagram of the complex interactions that are involved in the pathophysiology of HE is provided in Fig. 1. 
Jaeger V. et al: Astrocytes and microglia in encephalopathy

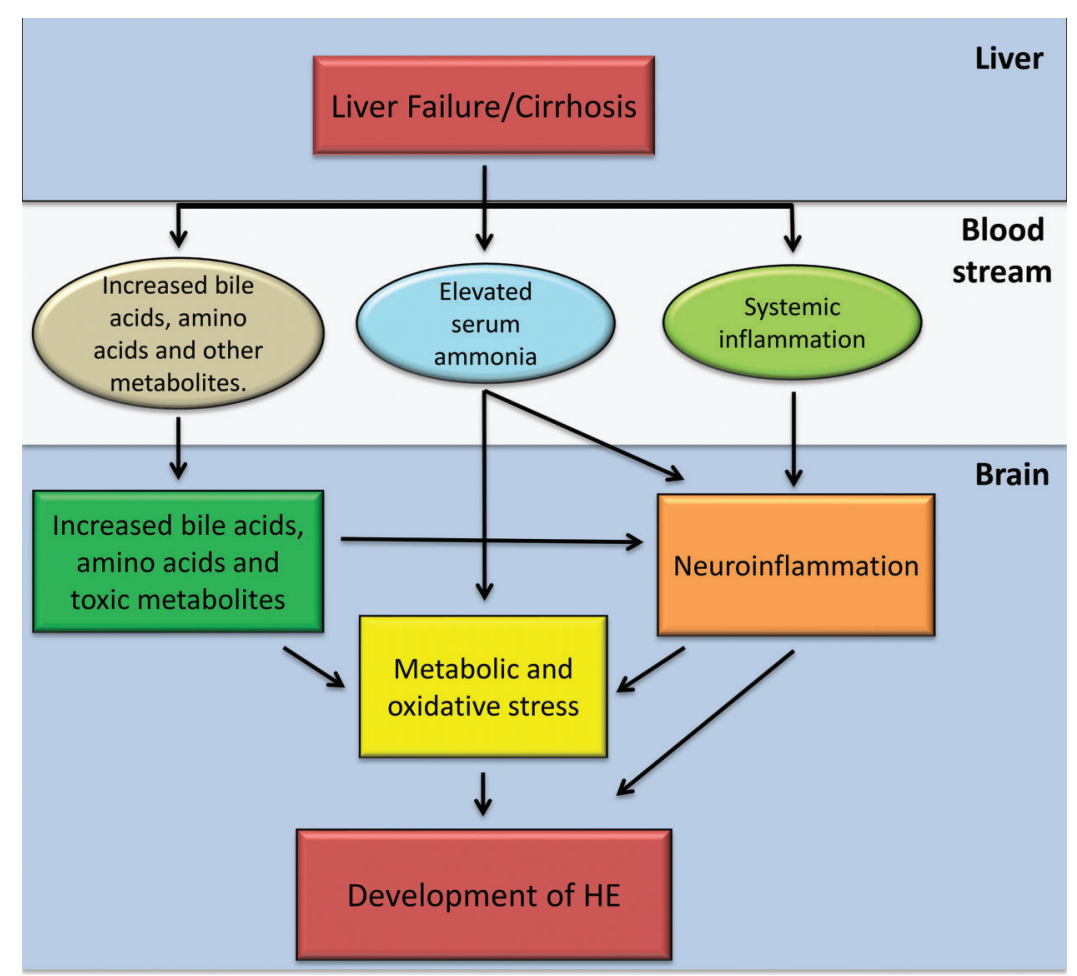

Fig. 1. Pathophysiology of HE. Following the development of liver failure or decompensated liver cirrhosis, there is an increase of circulating bile acids, amino acids, serum ammonia, and toxic metabolites as well as an increase of systemic inflammation. The systemic elevation of these factors leads to an increase of their concentrations in the brain, leading to metabolic and oxidative stress as well as increased neuroinflammation. Elevation of circulating cytokines and chemokines is associated with increased activation of microglia in the brain and subsequent neuroinflammation. The increase of neuroinflammation as well as the metabolic and oxidative stress present under these conditions promote the development of $\mathrm{HE}$.

\section{Astrocyte dysfunction}

Astrocytes are the most abundant glial cell in the CNS and are responsible for trophic and metabolic support of neurons and neuronal-glial communication, and are essential for bloodbrain barrier function. ${ }^{29}$ In response to increased ammonia concentrations during $\mathrm{HE}$, astrocytes undergo a change in morphology, developing large pale nuclei known as Alzheimer Type II astrocytosis. ${ }^{30}$ A large number of Alzheimer Type II astrocytic nuclei have been detected by autoradiography in hyperammonemic rats induced by methionine sulfoxamine. ${ }^{30}$ Hyperammonemia also leads to increased production of glutamine in astrocytes, resulting in increased osmotic pressure via astrocyte swelling. Swelling of astrocytes can lead to cerebral edema and a worsening of HE pathology. Research to date involving astrocytes has primarily focused on the effects of $\mathrm{HE}$ on regulation of oxidative stress, glutamine and glutamine synthase, brain water balance, and lactate metabolism.

\section{Glial fibrillary acidic protein (GFAP) and oxidative stress}

GFAP is an intermediate filament found in astrocytes that is used for both motility and maintenance of structural stability. ${ }^{31}$ Multiple studies have shown that GFAP is decreased during acute liver failure, thereby influencing the morphology and function of astrocytes. ${ }^{32,33}$ Investigating individual brain regions found that GFAP expression was greatly decreased in the corpus callosum ${ }^{34}$ and in the hippocampus during HE. ${ }^{35}$
GFAP has been shown to be important for cell volume regulation and therefore could contribute to the development of cerebral edema during HE. This has been demonstrated by the presence of increased brain water content when there was reduced GFAP protein levels during HE. ${ }^{32}$ A proposed mechanism for the down-regulation of GFAP during $\mathrm{HE}$ is that ammonia can interfere with metabolism, leading to decreased ATP levels and thus reducing GFAP production. ${ }^{36}$ In support of ammonia inhibiting GFAP expression, cultured astrocytes treated with ammonium chloride showed an $85 \%$ reduction in GFAP mRNA. ${ }^{37}$ GFAP expression has also been shown to be reduced during chronic liver disease and $\mathrm{HE}^{38,39}$ Loss of GFAP in chronic hepatitis was found to be related to astrocyte swelling, with subsequent loss of their stellate shape. ${ }^{40}$ Similarly, in bile duct-ligated (BDL) rats, altered astrocytes have been found but with no changes in the total number of astrocytes themselves. ${ }^{41}$

Studies have shown a direct relationship between astrocyte swelling and oxidative stress with p47phox-dependent activation of NADPH oxidase, contributing to reactive oxygen species generation from ammonia, in cultured rat astrocytes and cortical mouse brain slices. ${ }^{42}$ Oxidative and nitrosative stress can lead to an induction of astrocyte swelling. However, recent studies have shown the reverse is also true, as astrocyte swelling induces oxidative and nitrosative stress-creating a self-amplifying cycle. ${ }^{43}$ Hyperammonemia can lead to both oxidative and nitrosative stress, causing protein modifications such as nitration of tyrosine residues along with oxidation of RNA. ${ }^{44}$ That being said, oxidative 
stress, astrocyte swelling and down-regulation of GFAP likely contribute together to induce HE symptomology. Treatments aimed at reducing oxidative stress in astrocytes, such as treating cells with curcumin, reduce mitochondrial dysfunction and lead to increased GFAP levels. ${ }^{45}$ Therefore, many of the studies that are tying oxidative stress to changes in astrocyte swelling in the context of HE could be a result of the down-regulation of GFAP due to oxidative stress.

\section{Glutamine \& glutamine synthetase}

Currently, astrocytes are considered the primary target of ammonia toxicity because they are responsible for ammonia metabolism in the CNS via glutamine synthetase. ${ }^{46}$ Ammonia can freely cross the blood-brain barrier as perivascular processes of astrocytes rapidly metabolize ammonia from the circulation to prevent neurotoxicity using glutamine synthetase. ${ }^{47}$ Currently, few reports describe investigations into the role of glutamine synthetase on progression of $\mathrm{HE}$ due to acute liver failure.

One study using the acute liver failure model of lipopolysaccharide (LPS) and D-galactosamine injection determined that administration of methionine sulfoximine, a glutamine synthetase inhibitor, led to improved survival and reduced plasma tumor necrosis factor alpha (TNF $\alpha$ ) and interferon gamma levels. ${ }^{48}$ However, the effect of chronic HE on glutamine synthetase has been more extensively studied and the results are inconsistent with each other. After 4-week portacaval anastomosis, glutamine synthetase activity was significantly decreased in the hippocampus, cerebellum, and cerebral cortex but unchanged in other brain regions. ${ }^{49}$ Consistent with these findings, portacaval anastomosis rats have a $15 \%$ decrease in glutamine synthetase activity in the cerebral cortex. ${ }^{50}$ In addition, portacaval anastomosis rats at 6 months had regional variation of glutamine synthetase activity as well as a strong increase in glutamine synthetase in astrocyte end-feet processes. ${ }^{51}$ This regional variation was also shown in BDL rats 30 days after surgery, where glutamine synthetase activity was significantly decreased in the liver but there was no change in the frontal cortex. ${ }^{52}$ In addition, the administration of ornithine phenylacetate to reduce ammonia concentrations significantly reduced glutamine synthetase activity in the frontal cortex in BDL rats, indicating that treatments aimed at reducing ammonia may influence glutamine or glutamine synthetase activity. ${ }^{52}$ In postmortem cortical brain tissue from cirrhotic patients with and without $\mathrm{HE}$, glutamine synthase activity was significantly decreased whereas glutamine synthetase protein expression was not affected, which the authors surmise was a consequence of tyrosine nitration of the enzyme. ${ }^{53}$ This is supported, as cultured astrocytes treated with ammonium chloride have reduced glutamine synthetase activity and increased tyrosine nitration of glutamine synthetase. ${ }^{54}$

The elevation of astrocyte glutamine due to increased ammonia concentrations can lead to changes in brain water content. Glutamine is osmotically active and is a cause of brain edema and increased intracranial pressure. ${ }^{55}$ Elevated glutamine inside of astrocytes creates a hypertonic state leading to increased water accumulation in astrocytes and cytotoxic astrocyte swelling. In addition, glutamine enters the mitochondria of astrocytes where it is metabolized, yielding glutamate and ammonia. ${ }^{56}$ The mitochondrial compartment is small, and the high levels of ammonia that accumulate can lead to mitochondrial permeability transition pore opening, subsequent oxidative stress, and further astrocyte swelling. ${ }^{56,57}$

\section{Aquaporin-4 (AQP-4)}

AQP-4 is a bidirectional transmembrane water channel protein found on astrocyte end-feet and it plays a role in maintaining brain water homeostasis. ${ }^{58,59}$ Direct evidence for a causal role of AQP-4 in brain edema has been demonstrated in AQP-4 knockout mice. ${ }^{60}$ Studies have also shown the time course of cerebral edema development differs among brain regions, reflecting differences in AQP-4 distribution. ${ }^{59}$ Similarly, silencing AQP-4 gene expression in cultured astrocytes was shown to reduce water permeability under hypoosmotic conditions. ${ }^{61}$

AQP-4 is dysregulated during acute liver failure and HE. Acute liver failure is associated with up-regulation of AQP-4, as evidenced in thioacetamide-treated rats $^{62,63}$ as well as during galactosamine and LPS-induced liver failure. ${ }^{64}$ This phenomenon is also present in patients, as there was an upregulation of AQP-4 expression in the cerebral cortex from 8 patients with acute liver failure. ${ }^{33}$ In thioacetamide-treated rats, AQP-4 expression was positively correlated with brain water content. ${ }^{63}$ Exposure of rat cortical astrocytes cultures to interleukin (IL)-1 $\beta$ but not ammonia resulted in up-regulation of AQP-4, showing that brain edema may be worsened by inflammation. ${ }^{65}$

During chronic liver disease it was thought that cerebral edema was not a pathological characteristic of $H E$, as increased intracranial pressure is rare in this patient population. In addition, there is a decrease in grey matter and an increase in white matter in patients with cirrhosis, ${ }^{66}$ and low grade cerebral edema has been observed in patients with cirrhosis and minimal HE. ${ }^{67}$ In 4-week BDL rats, there is an increase of AQP-4 expression in the cortex, hippocampus, striatum and cerebellum that was associated with increased water content in all brain regions. ${ }^{68}$ This was validated in 4week BDL rats, that also had elevations of AQP-4 in the cortex. ${ }^{69}$ Interestingly, these researchers also used a galactosamine and high ammonia diet model in rats where AQP-4 was not changed following LPS injection but brain water percentage was significantly increased. ${ }^{69}$ Therefore, there may be other factors outside AQP-4 that need to be better characterized to understand the control of brain water content during chronic liver disease.

\section{Lactate}

Lactate is made in every cell from pyruvate, following a reaction catalyzed by lactate dehydrogenase. The liver will metabolize lactate into glucose, where it can serve as an energy source for all organs. In the brain, neurons use lactate as their preferred oxidative energy source and lactate is primarily synthesized by astrocytes, where it can be transported to neurons via the astrocyte-neuron lactate shuttle. ${ }^{70}$ In $\mathrm{HE}$, there is possible derangement of this relationship leading to hyperlactatemia. Increased concentrations of lactate, considered a marker of energy failure, are a mechanism of generating brain edema and neuronal dysfunction during HE. ${ }^{71}$ Lactate can induce swelling of cultured and primary astrocytes, as determined through in vitro studies. $^{72}$ However, lactate has been associated with increased brain edema during acute liver failure in patients and rodents and during chronic liver disease in BDL rats. ${ }^{73-75}$ 
Jaeger V. et al: Astrocytes and microglia in encephalopathy

Hyperlactatemia has been suggested as a prognostic marker of acetaminophen-induced acute liver failure, as increased arterial lactate correlated with the severity of $\mathrm{HE}$ and was present at significantly higher concentrations in nonsurvivors. ${ }^{76}$ The use of ${ }^{1} \mathrm{H}$ and ${ }^{13} \mathrm{C}$ NMR spectroscopy on the frontal cortex of rats with acute liver failure secondary to hepatic devascularization determined that lactate was increased $169.2 \%$ compared to controls. ${ }^{77}$ Likewise, use of hepatic devascularization to model acute liver failure determined that there were significant increases in lactate levels, with a $166 \%$ increase at $6 \mathrm{~h}$ and an increase in 3293\% at coma. ${ }^{78}$ Also, nuclear magnetic resonance spectroscopy was used to examine lactate usage by cells and determined that increased brain lactate synthesis along with impaired glucose oxidation were the major contributing factors to brain edema rather than accumulation of intracellular glutamine. ${ }^{74}$ It should be mentioned that not every study investigating lactate has found increases, as the use of ${ }^{1} \mathrm{H}$ and ${ }^{31} \mathrm{P}$ magnetic resonance spectroscopy found essentially no change of brain lactate in BDL rats at 4 weeks or 8 weeks following surgery. ${ }^{79}$

\section{Microglia activation}

Microglia are cells of myeloid origin, whose main function is to control the immune response of the CNS. ${ }^{80}$ Additionally, activated microglia are known to induce the inflammatory response in the brain by releasing proinflammatory cytokines, such as IL- $1 \alpha$, IL- $1 \beta$ and TNF $\alpha .{ }^{81}$ Evidence of neuroinflammation has been shown in $\mathrm{HE}$ patients with acute liver failure and chronic liver disease. In patients with acute liver failure, microglia activation occurs as shown by increased immunostaining for human leukocyte antigen DR (CR3/43) when compared to controls. ${ }^{21}$ In post-mortem cortical brain tissue from patients with liver cirrhosis and overt $\mathrm{HE}$, there is up-regulation of the microglia marker ionized calcium binding adaptor molecule 1 (known as IBA1) when compared to cirrhotic patients without HE. ${ }^{82}$

Multiple reports have shown microglia activation in the $\mathrm{BDL}$ model of chronic HE. ${ }^{34,83,84}$ Interestingly, one study found that BDL triggered alternative activation of microglia. ${ }^{34}$ Instead of the classical microglial markers OX6, ED1 and IBA1 along with pro-inflammatory markers IL-1 $\beta$ and inducible nitric oxide synthase were not elevated but transforming growth factor beta 1 (known as TGF $\beta 1$ ) was found to be increased. ${ }^{34}$ Another study using post-mortem tissue from cirrhotic patients with HE observed activated microglia with hypertrophied cell bodies and thickened processes along with higher levels of IL- $6 .^{85}$ Outside of cytokines, microglia activation can be assessed by ${ }^{11} \mathrm{C}-\mathrm{PK} 11195$, which is a positive emission tomography ligand for translocator protein. ${ }^{86}$ In the context of acute $\mathrm{HE},{ }^{11} \mathrm{C}-\mathrm{PK} 1195$ and ${ }^{18} \mathrm{~F}-\mathrm{DPA}-714$ have been used and found to detect neuroinflammation in thioacetamide-treated rats by binding to translocator protein. ${ }^{87}$ Interestingly, translocator protein has been deleted from astrocytes, demonstrating an increase of mitochondria permeability transition and cell volume in response to ammonia, indicating that this protein is involved in more processes than just neuroinflammation. ${ }^{88}$

That being said, not all evidence shows induction of a proinflammatory phenotype during $\mathrm{HE}$ as microglia polarization occurs in cirrhotic patients, with both pro-inflammatory M1 and anti-inflammatory M2 phenotypes being present. $^{89}$ Research involving microglia has primarily focused on signals leading to their activation, chemokine and cytokine regulation, and oxidative stress.

\section{Ammonia and microglia}

Studies have investigated if hyperammonemia causes microglia activation in both acute and chronic HE. The exposure of primary cell cultures of microglia to ammonia led to an increase in both synthesis and release of IL- 6 and TNF $\alpha$ compared to basal microglia. ${ }^{90}$ Likewise, in the azoxymethane model of acute liver failure, the investigators found microglia to be activated; however, they found microglia to not be activated in mice injected with ammonium chloride. ${ }^{91}$ In rats fed an ammonia-containing diet for 4 weeks to induce a state of hyperammonemia, microglia activation was observed in the hippocampus, that could be reversed by removing the ammonia-containing diet for 2 or 4 weeks. ${ }^{92}$ Interestingly, in a co-culture of rat astrocytes and microglia that were treated with ammonium chloride and LPS, it was found that ammonia treatment did not up-regulate the gene expression of IL- $1 \alpha$, IL- $1 \beta$, IL- 6 or TNF $\alpha$ in microglia or cocultured astrocytes and microglia. ${ }^{93}$ The investigators also found that astrocytes reduced the up-regulation of microglia activation markers induced by LPS. ${ }^{93}$ As elevated brain ammonia concentrations and neuroinflammation are pathological characteristics of $\mathrm{HE}$, it is evident that gaining greater understanding into the exact influences of ammonia on microglia activation in the different contexts of $\mathrm{HE}$ are necessary.

\section{Chemokines}

Outside of ammonia, chemokines are the primary contributors to activating microglia during states of neuroinflammation. Chemokines are involved with cell-cell communication and regulate neuroinflammation by influencing migration and activation of immune cells. ${ }^{94}$ Most studies have focused on investigating the pro-inflammatory chemokine ligand 2 (CCL2) and anti-inflammatory C-X3-C ligand 1 (CX3CL1). In mice that had undergone bile duct ligation, the release of CCL2 in the brain triggered recruitment of infiltrating monocytes, leading to neurological decline. ${ }^{83}$ The investigators also found that microglia have increased levels of CCL2 and intraperitoneal injection of anti-TNF $\alpha$ serum led to reduced CCL2 expression in microglia. ${ }^{83}$ In a model of portal hypertension using triple calibrated portal vein ligation for 1 month in rats, the investigators found that the CX3CL1 expression was not changed in the hippocampus or cerebellum, but its receptor CX3CR1 was significantly up-regulated in both regions while stromal cell-derived factor 1 alpha and C-X-C chemokine receptor type 4 were up-regulated in only the hippocampus. ${ }^{95}$ Specific targeting of CCL2 activity through intraperitoneal injection of chemokine receptor 2 and chemokine receptor 4 inhibitors during acute liver failure in azoxymethane- or thioacetamide-treated mice was found to reduce microglia activation and improve neurological function. ${ }^{96,97}$ In the azoxymethane model of acute liver failure, injection of soluble CX3CL1 was found to reduce microglia activation and improve time taken to reach coma, indicating that an imbalance of CCL2 and CX3CL1 expression may be driving the activation of microglia during HE. ${ }^{98}$ 


\section{Cytokines}

In patients and animal models of $\mathrm{HE}$, systemic inflammation causes worsening of neurological function and it has been proposed that pro-inflammatory signals act in concert with ammonia to generate the neurological complications of acute liver failure and chronic liver disease. ${ }^{21,99,100} \mathrm{TNF} \alpha$ is a potent pro-inflammatory cytokine that has been shown to activate microglia in a number of experimental models of neuroinflammation. ${ }^{101,102}$ Circulating levels of TNF $\alpha$ are increased as a function of the severity of $\mathrm{HE}$ in both patients ${ }^{103}$ and experimental animals ${ }^{104}$ with liver failure. Moreover, the presence of TNF $\alpha$ gene polymorphisms is known to influence the outcomes of patients with acute liver failure. ${ }^{105}$ During $\mathrm{HE}$, systemic levels of $\mathrm{TNF}_{\alpha}$ are increased in the azoxymethane model of acute liver failure. ${ }^{106}$ Inhibition of TNF $\alpha$ signaling by systemic treatment with etanercept reduced systemic inflammation, attenuated the neurological decline, and prevented microglial activation in the cerebral cortex. ${ }^{106}$ These data support the hypothesis that peripherally-derived $\mathrm{TNF} \alpha$, at least in part, contributes to the microglial activation and subsequent neurological decline of liver failure. In further support of this concept, neurological complications occurring in $\mathrm{BDL}$ mice were shown to be the consequence of monocyte recruitment in response to $\mathrm{TNF} \alpha$ signaling and to occur via microglial activation. ${ }^{83}$ Specifically, peripheral TNF $\alpha$ signaling stimulates microglia to produce CCL2, which subsequently mediates monocyte recruitment into the brain. ${ }^{83}$

Pro-inflammatory mediators other than TNF $\alpha$ can contribute to $\mathrm{HE}$ pathogenesis as well. IL- $1 \beta$ and microglia activation are increased in rats after portacaval shunt and administering sildenafil was found to reduce neuroinflammation and microglia activation. ${ }^{107}$ In azoxymethane-treated mice, cortex IL-1 $\beta$, IL-6, TNF $\alpha$ and CCL2 protein expression were increased. ${ }^{108}$ The use of anti-TGF $\beta 1$ antibodies or genetic ablation of transforming growth factor beta receptor 2 (known as TGF $\beta$ R2) in neurons of azoxymethane-treated mice led to reduced microglia activation and normalized levels of IL-1 $\beta, \mathrm{IL}-6, \mathrm{TNF} \alpha$ and $\mathrm{CCL} 2$, giving support that TGF $\beta$ R2-mediated signaling contributes to neuroinflammation during HE. ${ }^{108}$ Interestingly, the use of the gamma aminobutyric acid antagonist bicuculine was found to reduce the expression of IL-1 $\beta$, but not effect microglia activation in ammonium chloride-fed rats. ${ }^{109}$ This gives support that the pro-inflammatory state during elevated levels of ammonia in the brain may not be directly linked to microglia activation, though more studies are need in this area to better characterize this.

\section{Clinical management of HE}

The most efficacious treatment for patients with HE is liver transplantation, with cognitive measures and metabolites in the brain becoming normalized after transplantation. ${ }^{110}$ That being said, in comparison to cirrhotic patients without HE that undergo liver transplantation, those with HE that undergo liver transplantation have impaired cognitive function, with one study showing that $13 \%$ of the cohort of patients maintained mild cognitive impairment 6-12 months after liver transplantation. ${ }^{111}$ Besides liver transplantation, there are therapeutics commonly used, including lactulose or nonabsorbable antibiotics such as rifaximin. Lactulose is a nondigestible disaccharide that is metabolized by bacteria in the colon, decreasing $\mathrm{pH}$, reducing bacterial ammonia production,

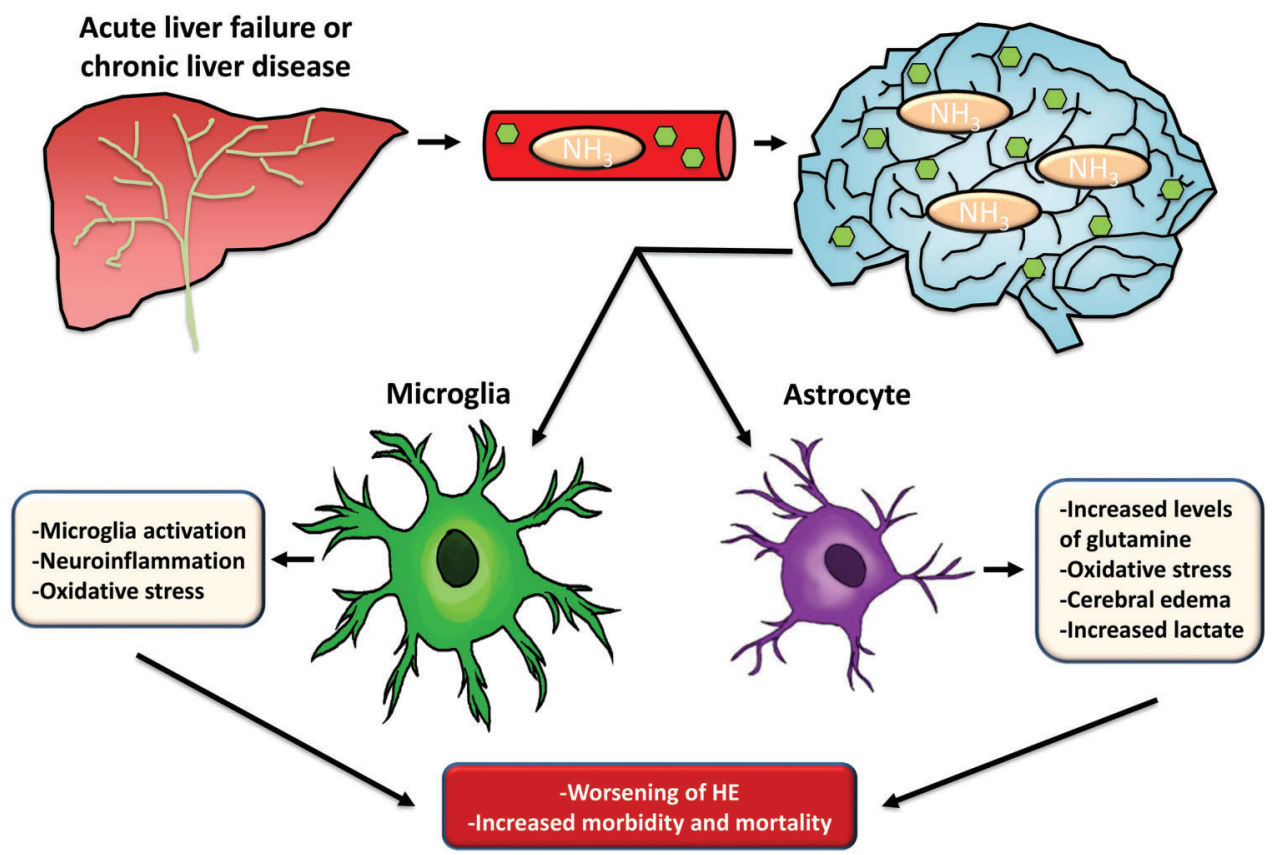

Fig. 2. Summary of the involvement of microglia and astrocytes in HE pathology. The liver can lose its ability to function during acute liver failure or chronic liver disease. When this occurs, toxins, such as ammonia, enter the circulation and can enter the brain. After accumulating in neural tissue, they cause a disruption of astrocyte and microglia cellular function. Astrocytes metabolize ammonia, leading to an increase of glutamine, cell swelling, cerebral edema, oxidative stress and hyperlactatemia. Microglia become activated leading to increased neuroinflammation due to pro-inflammatory cytokine release and oxidative stress. Together, these changes in neural cellular function lead to increased HE pathology, resulting in increased morbidity and mortality.

Abbreviations: $\mathrm{HE}$, hepatic encephalopathy; $\mathrm{NH}_{3}$, ammonia. 
and producing more ammonium from ammonia, which cannot be absorbed into the intestine. ${ }^{112}$ Rifaximin is used to eliminate bacteria with a specific efficacy for anaerobic bacteria and can reduce ammonia production, endotoxin generation, and inflammation. ${ }^{113}$ There are a variety of other treatments that have been investigated, including L-ornithine-L-aspartate, ornithine phenylacetate, glycerol phenylbutyrate, various probiotics, AST-120 and others that are generally aimed at reducing ammonia levels in HE patients that have had limited improvement when compared to lactulose or rifaximin. ${ }^{5,114}$

Other than these direct treatments of $\mathrm{HE}$, clinical management strategies for HE patients include alleviating precipitating factors that contribute to the syndrome and management of comorbidities. In HE patients that develop hepatorenal syndrome there is a complete disruption of the urea cycle, leading to even greater concentrations of ammonia in the circulation due to both loss of hepatic function and kidney failure. Other conditions can lead to inhibition of ammonia metabolism, including sarcopenia in cirrhotic patients, as these have reduced ability to metabolize ammonia into glutamine due to muscle loss. The presence of sarcopenia is associated with development of both minimal and overt HE. ${ }^{115}$ Infections are also a risk factor for HE with bacterial overgrowth, spontaneous bacterial peritonitis, and other infections contributing to morbidity and HE progression. ${ }^{116}$ Controlling these systemic complications that contribute to $\mathrm{HE}$ while lowering circulating ammonia concentrations are the most effective strategies currently used for management of this syndrome.

\section{Summary and future perspectives}

HE is a devastating consequence of acute liver failure and chronic liver disease that is associated with increased morbidity and mortality and has few effective treatments besides liver transplantation. The elevation of circulating ammonia levels that occurs following the disruption of the urea cycle leads to accumulation of ammonia in astrocytes. This generates a myriad of complications, including dysregulation of glutamine concentrations, elevation of lactate, generation of oxidative and nitrosative stress, and cerebral edema. In addition, hepatic injury and loss of liver function leads to systemic inflammation, which results in activation of microglia in the brain due to both increases of ammonia and dysregulation of chemokine concentrations. This activation of microglia leads to the production of pro-inflammatory cytokines that ultimately work in concert with astrocyte dysfunction to further increase oxidative stress and cerebral edema. Collectively, this results in increased cerebral edema, intracranial pressure, and worse HE pathology. A working model of these aspects of HE pathology are presented in Fig. 2. Ultimately, if we are to develop new treatments for the management of $\mathrm{HE}$, gaining a greater understanding into the specific cellular contributions to neurological dysfunction associated with HE is necessary to identify new targets that allow for better management of this syndrome.

\section{Acknowledgments}

This study was funded by a VA Career Development award (BX003486) from the United States Department of Veterans Affairs Biomedical Laboratory Research and Development Service to Dr. Matthew McMillin. This study was also funded by NIH R01 awards (DK082435 and DK112803) and a VA Merit award (BX002638) from the United States Department of Veterans Affairs Biomedical Laboratory Research and Development Service to Dr. Sharon DeMorrow. This work was also supported by the resources of the Central Texas Veterans Health Care System Research Service, Temple, Texas. The content is the responsibility of the author(s) alone and does not necessarily reflect the views or policies of the Department of Veterans Affairs or the United States Government.

\section{Conflict of interest}

The authors have no conflict of interests related to this publication.

\section{Author contributions}

Contributed to draft and critically revise the document (VJ, $\mathrm{SD})$, draft the document, create the figures, and critically revise the document (MM). All authors approved the final version of this manuscript.

\section{References}

[1] Martino ME, Fernández-Lorente J, Romero-Vives M, Bárcena R, Gaztelu JM. Brain oscillatory activity during sleep shows unknown dysfunctions in early encephalopathy. J Physiol Biochem 2014;70:821-835. doi: 10. 1007/s13105-014-0351-2.

[2] Nardelli S, Allampati S, Riggio O, Mullen KD, Prakash R, Gioia S, et al. Hepatic encephalopathy is associated with persistent learning impairments despite adequate medical treatment: A multicenter, international study. Dig Dis Sci 2017;62:794-800. doi: 10.1007/s10620-016-4425-6.

[3] Hirode G, Vittinghoff E, Wong RJ. Increasing burden of hepatic encephalopathy among hospitalized adults: An analysis of the 2010-2014 national inpatient sample. Dig Dis Sci 2019;64:1448-1457. doi: 10.1007/s10620019-05576-9.

[4] Suraweera D, Sundaram V, Saab S. Evaluation and management of hepatic encephalopathy: Current status and future directions. Gut Liver 2016;10: 509-519. doi: 10.5009/gnl15419.

[5] Patidar KR, Bajaj JS. Covert and overt hepatic encephalopathy: Diagnosis and management. Clin Gastroenterol Hepatol 2015;13:2048-2061. doi: $10.1016 /$ j.cgh.2015.06.039.

[6] Dharel N, Bajaj JS. Definition and nomenclature of hepatic encephalopathy. J Clin Exp Hepatol 2015;5:S37-S41. doi: 10.1016/j.jceh.2014.10.001.

[7] Grover VP, Tognarelli JM, Massie N, Crossey MM, Cook NA, Taylor-Robinson SD. The why and wherefore of hepatic encephalopathy. Int J Gen Med 2015; 8:381-390. doi: 10.2147/IJGM.S86854.

[8] NeSmith M, Ahn J, Flamm SL. Contemporary understanding and management of overt and covert hepatic encephalopathy. Gastroenterol Hepatol (N Y) 2016;12:91-100.

[9] Liu A, Perumpail RB, Kumari R, Younossi ZM, Wong RJ, Ahmed A. Advances in cirrhosis: Optimizing the management of hepatic encephalopathy. World J Hepatol 2015;7:2871-2879. doi: 10.4254/wjh.v7.i29.2871.

[10] Bernal W, Auzinger G, Dhawan A, Wendon J. Acute liver failure. Lancet 2010;376:190-201. doi: 10.1016/S0140-6736(10)60274-7.

[11] Bustamante J, Rimola A, Ventura PJ, Navasa M, Cirera I, Reggiardo V, et al. Prognostic significance of hepatic encephalopathy in patients with cirrhosis. J Hepatol 1999;30:890-895. doi: 10.1016/s0168-8278(99)80144-5.

[12] Romeiro FG, Augusti L. Nutritional assessment in cirrhotic patients with hepatic encephalopathy. World J Hepatol 2015;7:2940-2954. doi: 10. 4254/wjh.v7.i30.2940.

[13] Hakvoort TB, He Y, Kulik W, Vermeulen JL, Duijst S, Ruijter JM, et al. Pivotal role of glutamine synthetase in ammonia detoxification. Hepatology 2017; 65:281-293. doi: 10.1002/hep.28852.

[14] Hansen BA, Vilstrup H. Increased intestinal hydrolysis of urea in patients with alcoholic cirrhosis. Scand J Gastroenterol 1985;20:346-350. doi: 10. 3109/00365528509091662.

[15] Sørensen M. Update on cerebral uptake of blood ammonia. Metab Brain Dis 2013;28:155-159. doi: 10.1007/s11011-013-9395-1.

[16] Clemmesen JO, Kondrup J, Ott P. Splanchnic and leg exchange of amino acids and ammonia in acute liver failure. Gastroenterology 2000;118: 1131-1139. doi: 10.1016/s0016-5085(00)70366-0. 
[17] Riggio O, Nardelli S, Pasquale C, Pentassuglio I, Gioia S, Onori E, et al. No effect of albumin infusion on the prevention of hepatic encephalopathy after transjugular intrahepatic portosystemic shunt. Metab Brain Dis 2016;31: 1275-1281. doi: 10.1007/s11011-015-9713-x.

[18] Clària J, Stauber RE, Coenraad MJ, Moreau R, Jalan R, Pavesi M, et al. Systemic inflammation in decompensated cirrhosis: Characterization and role in acute-on-chronic liver failure. Hepatology 2016;64:1249-1264. doi: 10 . 1002/hep. 28740.

[19] Moore JK, MacKinnon AC, Man TY, Manning JR, Forbes SJ, Simpson KJ. Patients with the worst outcomes after paracetamol (acetaminophen)induced liver failure have an early monocytopenia. Aliment Pharmacol Ther 2017;45:443-454. doi: 10.1111/apt.13878.

[20] Dirchwolf M, Podhorzer A, Marino M, Shulman C, Cartier M, Zunino M, et al. Immune dysfunction in cirrhosis: Distinct cytokines phenotypes according to cirrhosis severity. Cytokine 2016;77:14-25. doi: 10.1016/j.cyto.2015. 10.006 .

[21] Butterworth RF. Hepatic encephalopathy: a central neuroinflammatory disorder? Hepatology 2011;53:1372-1376. doi: 10.1002/hep.24228.

[22] Record CO, Buxton B, Chase RA, Curzon G, Murray-Lyon IM, Williams R. Plasma and brain amino acids in fulminant hepatic failure and their relationship to hepatic encephalopathy. Eur J Clin Invest 1976;6:387-394. doi: 10. 1111/j.1365-2362.1976.tb00533.x.

[23] Bron B, Waldram R, Silk DB, Williams R. Serum, cerebrospinal fluid, and brain levels of bile acids in patients with fulminant hepatic failure. Gut 1977;18:692-696. doi: 10.1136/gut.18.9.692.

[24] Spahr L, Butterworth RF, Fontaine S, Bui L, Therrien G, Milette PC, et al. Increased blood manganese in cirrhotic patients: relationship to pallidal magnetic resonance signal hyperintensity and neurological symptoms. Hepatology 1996;24:1116-1120. doi: 10.1002/hep.510240523.

[25] Pomier-Layrargues G, Spahr L, Butterworth RF. Increased manganese concentrations in pallidum of cirrhotic patients. Lancet 1995;345:735. doi: 10. 1016/s0140-6736(95)90909-5.

[26] Butterworth RF, Spahr L, Fontaine S, Layrargues GP. Manganese toxicity, dopaminergic dysfunction and hepatic encephalopathy. Metab Brain Dis 1995;10:259-267. doi: 10.1007/bf02109357.

[27] Sharma P, Sharma BC. Management of overt hepatic encephalopathy. J Clin Exp Hepatol 2015;5:S82-S87. doi: 10.1016/j.jceh.2014.04.004.

[28] Sawhney R, Holland-Fischer P, Rosselli M, Mookerjee RP, Agarwal B, Jalan R. Role of ammonia, inflammation, and cerebral oxygenation in brain dysfunction of acute-on-chronic liver failure patients. Liver Transpl 2016;22:732742. doi: $10.1002 /$ It. 24443

[29] Blackburn D, Sargsyan S, Monk PN, Shaw PJ. Astrocyte function and role in motor neuron disease: a future therapeutic target? Glia 2009;57:12511264. doi: 10.1002/glia.20848.

[30] Brumback RA, Lapham LW. DNA synthesis in Alzheimer type II astrocytosis. The question of astrocytic proliferation and mitosis in experimentally induced hepatic encephalopathy. Arch Neurol 1989;46:845-848. doi: 10 . 1001/archneur.1989.00520440027016.

[31] Butterworth RF. Pathogenesis of hepatic encephalopathy and brain edema in acute liver failure. J Clin Exp Hepatol 2015;5:S96-S103. doi: 10.1016/j. jceh.2014.02.004.

[32] Bélanger M, Desjardins P, Chatauret N, Butterworth RF. Loss of expression of glial fibrillary acidic protein in acute hyperammonemia. Neurochem Int 2002;41:155-160. doi: 10.1016/s0197-0186(02)00037-2.

[33] Thumburu KK, Dhiman RK, Vasishta RK, Chakraborti A, Butterworth RF, Beauchesne $E$, et al. Expression of astrocytic genes coding for proteins implicated in neural excitation and brain edema is altered after acute liver failure. J Neurochem 2014;128:617-627. doi: 10.1111/jnc.12511.

[34] Wright GA, Sharifi Y, Newman TA, Davies N, Vairappan B, Perry HV, et al. Characterisation of temporal microglia and astrocyte immune responses in bile duct-ligated rat models of cirrhosis. Liver Int 2014;34:1184-1191. doi: 10.1111/liv.12481.

[35] Suarez I, Bodega G, Fernandez B. Changes in glial fibrillary acidic protein immunoreactivity in response to experimental hepatic encephalopathy in the rat hippocampus. Restor Neurol Neurosci 1998;12:49-54.

[36] Haghighat N, McCandless DW, Geraminegad P. Responses in primary astrocytes and C6-glioma cells to ammonium chloride and dibutyryl cyclic-AMP. Neurochem Res 2000;25:277-284. doi: 10.1023/a:1007535922977.

[37] Neary JT, Whittemore SR, Zhu Q, Norenberg MD. Destabilization of glial fibrillary acidic protein mRNA in astrocytes by ammonia and protection by extracellular ATP. J Neurochem 1994;63:2021-2027. doi: 10.1046/j.14714159.1994.63062021.x.

[38] Arias N, Méndez M, Alcalde I, Íñigo-Portugués A, Merayo-Lloves J, Arias J, et al. Assessing the brain through the eye: New ways to explore hepatic encephalopathy. Physiol Behav 2017;173:263-271. doi: 10.1016/j. physbeh.2017.02.022.

[39] Arias N, Méndez M, Gómez-Lázaro E, Azpiroz A, Arias JL. Main target of minimal hepatic encephalopathy: Morphophysiological, inflammatory and metabolic view. Physiol Behav 2015;149:247-254. doi: 10.1016/j. physbeh.2015.06.019.
[40] Fomenko OZ, Ushakova HO, Piierzhynovs'kyĭ SH. Astroglia proteins in the rat brain in experimental chronic hepatitis and 2-oxoglutarate effect. Ukr Biokhim Zh (1999) 2011;83:69-76.

[41] Rivera-Mancía S, Montes S, Méndez-Armenta M, Muriel P, Ríos C. Morphological changes of rat astrocytes induced by liver damage but not by manganese chloride exposure. Metab Brain Dis 2009;24:243-255. doi: 10. 1007/s11011-009-9138-5.

[42] Reinehr R, Görg B, Becker S, Qvartskhava N, Bidmon HJ, Selbach O, et al. Hypoosmotic swelling and ammonia increase oxidative stress by NADPH oxidase in cultured astrocytes and vital brain slices. Glia 2007;55:758771. doi: $10.1002 /$ glia.20504.

[43] Lachmann V, Görg B, Bidmon HJ, Keitel V, Häussinger D. Precipitants of hepatic encephalopathy induce rapid astrocyte swelling in an oxidative stress dependent manner. Arch Biochem Biophys 2013;536:143-151. doi: 10.1016/j.abb.2013.05.004.

[44] Häussinger D, Görg B. Interaction of oxidative stress, astrocyte swelling and cerebral ammonia toxicity. Curr Opin Clin Nutr Metab Care 2010;13:87-92. doi: 10.1097/MCO.0b013e328333b829.

[45] Daverey A, Agrawal SK. Curcumin alleviates oxidative stress and mitochondrial dysfunction in astrocytes. Neuroscience 2016;333:92-103. doi: 10. 1016/j.neuroscience.2016.07.012.

[46] Bobermin LD, Wartchow KM, Flores MP, Leite MC, Quincozes-Santos A, Gonçalves CA. Ammonia-induced oxidative damage in neurons is prevented by resveratrol and lipoic acid with participation of heme oxygenase 1 . Neurotoxicology 2015;49:28-35. doi: 10.1016/j.neuro.2015.05.005.

[47] Suárez I, Bodega G, Fernández B. Glutamine synthetase in brain: effect of ammonia. Neurochem Int 2002;41:123-142. doi: 10.1016/s0197-0186 (02)00033-5.

[48] Jambekar AA, Palma E, Nicolosi L, Rasola A, Petronilli V, Chiara F, et al. A glutamine synthetase inhibitor increases survival and decreases cytokine response in a mouse model of acute liver failure. Liver Int 2011;31:12091221. doi: 10.1111/j.1478-3231.2011.02553.x.

[49] Girard G, Giguère JF, Butterworth RF. Region-selective reductions in activities of glutamine synthetase in rat brain following portacaval anastomosis. Metab Brain Dis 1993;8:207-215. doi: 10.1007/bf01001062.

[50] Butterworth RF, Girard G, Giguère JF. Regional differences in the capacity for ammonia removal by brain following portocaval anastomosis. J Neurochem 1988;51:486-490. doi: 10.1111/j.1471-4159.1988.tb01064.x.

[51] Suárez I, Bodega G, Arilla E, Fernández B. Region-selective glutamine synthetase expression in the rat central nervous system following portocaval anastomosis. Neuropathol Appl Neurobiol 1997;23:254-261. doi: 10. 1111/j.1365-2990.1997.tb01209.x.

[52] Jover-Cobos M, Noiret L, Lee K, Sharma V, Habtesion A, Romero-Gomez M, et al. Ornithine phenylacetate targets alterations in the expression and activity of glutamine synthase and glutaminase to reduce ammonia levels in bile duct ligated rats. J Hepatol 2014;60:545-553. doi: 10.1016/j.jhep. 2013.10.012

[53] Görg B, Qvartskhava N, Bidmon HJ, Palomero-Gallagher N, Kircheis G, Zilles $\mathrm{K}$, et al. Oxidative stress markers in the brain of patients with cirrhosis and hepatic encephalopathy. Hepatology 2010;52:256-265. doi: 10.1002/hep. 23656.

[54] Schliess F, Görg B, Fischer R, Desjardins P, Bidmon HJ, Herrmann A, et al. Ammonia induces MK-801-sensitive nitration and phosphorylation of protein tyrosine residues in rat astrocytes. FASEB J 2002;16:739-741. doi: 10.1096/fj.01-0862fje.

[55] Tofteng F, Hauerberg J, Hansen BA, Pedersen CB, Jørgensen L, Larsen FS. Persistent arterial hyperammonemia increases the concentration of glutamine and alanine in the brain and correlates with intracranial pressure in patients with fulminant hepatic failure. J Cereb Blood Flow Metab 2006;26: 21-27. doi: 10.1038/sj.jcbfm.9600168.

[56] Albrecht J, Norenberg MD. Glutamine: a Trojan horse in ammonia neurotoxicity. Hepatology 2006;44:788-794. doi: 10.1002/hep.21357.

[57] Norenberg MD, Rao KV, Jayakumar AR. Mechanisms of ammonia-induced astrocyte swelling. Metab Brain Dis 2005;20:303-318. doi: 10. 1007/s11011-005-7911-7.

[58] Nielsen S, Nagelhus EA, Amiry-Moghaddam M, Bourque C, Agre P, Ottersen OP. Specialized membrane domains for water transport in glial cells: highresolution immunogold cytochemistry of aquaporin-4 in rat brain. J Neurosci 1997;17:171-180. doi: 10.1523/JNEUROSCI.17-01-00171.1997.

[59] Amiry-Moghaddam M, Xue R, Haug FM, Neely JD, Bhardwaj A, Agre P, et al. Alpha-syntrophin deletion removes the perivascular but not endothelial pool of aquaporin-4 at the blood-brain barrier and delays the development of brain edema in an experimental model of acute hyponatremia. FASEB J 2004;18:542-544. doi: 10.1096/fj.03-0869fje.

[60] Manley GT, Binder DK, Papadopoulos MC, Verkman AS. New insights into water transport and edema in the central nervous system from phenotype analysis of aquaporin-4 null mice. Neuroscience 2004;129:983-991. doi: 10.1016/j.neuroscience.2004.06.088.

[61] Nicchia GP, Frigeri A, Liuzzi GM, Svelto M. Inhibition of aquaporin-4 expression in astrocytes by RNAi determines alteration in cell morphology, growth, 
and water transport and induces changes in ischemia-related genes. FASEB J 2003;17:1508-1510. doi: 10.1096/fj.02-1183fje.

[62] Yang JH, Song ZJ, Liao CD, You LY, Xu Y, Yang J, et al. The relationship of the expression of aquaporin-4 and brain edema in rats with acute liver failure. Zhonghua Gan Zang Bing Za Zhi 2006;14:215-216.

[63] Wang LQ, Zhu SM, Zhou HJ, Pan CF. Expression of aquaporin-4 during brain edema in rats with thioacetamide-induced acute encephalopathy. Zhonghua Yi Xue Za Zhi 2011;91:2573-2577. doi: 10.3760/cma.j.issn.03762491.2011.36.016.

[64] Eefsen M, Jelnes P, Schmidt LE, Vainer B, Bisgaard HC, Larsen FS. Brain expression of the water channels aquaporin-1 and -4 in mice with acute liver injury, hyperammonemia and brain edema. Metab Brain Dis 2010; 25:315-323. doi: 10.1007/s11011-010-9213-y.

[65] Chastre A, Jiang W, Desjardins P, Butterworth RF. Ammonia and proinflammatory cytokines modify expression of genes coding for astrocytic proteins implicated in brain edema in acute liver failure. Metab Brain Dis 2010;25: 17-21. doi: 10.1007/s11011-010-9185-y.

[66] Zhang LJ, Qi R, Zhong J, Xu Q, Zheng G, Lu GM. The effect of hepatic encephalopathy, hepatic failure, and portosystemic shunt on brain volume of cirrhotic patients: a voxel-based morphometry study. PLoS One 2012;7: e42824. doi: 10.1371/journal.pone.0042824.

[67] Rai R, Ahuja CK, Agrawal S, Kalra N, Duseja A, Khandelwal N, et al. Reversal of low-grade cerebral edema after lactulose/rifaximin therapy in patients with cirrhosis and minimal hepatic encephalopathy. Clin Transl Gastroenterol 2015;6:e111. doi: 10.1038/ctg.2015.38.

[68] Dhanda S, Sandhir R. Blood-brain barrier permeability is exacerbated in experimental model of hepatic encephalopathy via MMP-9 activation and downregulation of tight junction proteins. Mol Neurobiol 2018;55:36423659. doi: 10.1007/s12035-017-0521-7.

[69] Wright G, Soper R, Brooks HF, Stadlbauer V, Vairappan B, Davies NA, et al. Role of aquaporin-4 in the development of brain oedema in liver failure. J Hepatol 2010;53:91-97. doi: 10.1016/j.jhep.2010.02.020.

[70] Bélanger M, Magistretti PJ. The role of astroglia in neuroprotection. Dialogues Clin Neurosci 2009;11:281-295.

[71] Bosoi CR, Rose CF. Elevated cerebral lactate: Implications in the pathogenesis of hepatic encephalopathy. Metab Brain Dis 2014;29:919-925. doi: 10. 1007/s11011-014-9573-9.

[72] Staub F, Baethmann A, Peters J, Weigt H, Kempski O. Effects of lactacidosis on glial cell volume and viability. J Cereb Blood Flow Metab 1990;10:866876. doi: $10.1038 / \mathrm{jcbfm} .1990 .143$.

[73] Tofteng F, Jorgensen L, Hansen BA, Ott P, Kondrup J, Larsen FS. Cerebral microdialysis in patients with fulminant hepatic failure. Hepatology 2002; 36:1333-1340. doi: 10.1053/jhep.2002.36944.

[74] Zwingmann C, Chatauret N, Leibfritz D, Butterworth RF. Selective increase of brain lactate synthesis in experimental acute liver failure: results of a $[\mathrm{H}-\mathrm{C}]$ nuclear magnetic resonance study. Hepatology 2003;37:420-428. doi: 10.1053/jhep.2003.50052.

[75] Bosoi CR, Zwingmann C, Marin H, Parent-Robitaille C, Huynh J, Tremblay M, et al. Increased brain lactate is central to the development of brain edema in rats with chronic liver disease. J Hepatol 2014;60:554-560. doi: 10.1016/j. jhep.2013.10.011.

[76] Schmidt LE, Larsen FS. Prognostic implications of hyperlactatemia, multiple organ failure, and systemic inflammatory response syndrome in patients with acetaminophen-induced acute liver failure. Crit Care Med 2006;34: 337-343. doi: 10.1097/01.ccm.0000194724.70031.b6.

[77] Chatauret N, Zwingmann C, Rose C, Leibfritz D, Butterworth RF. Effects of hypothermia on brain glucose metabolism in acute liver failure: a H/Cnuclear magnetic resonance study. Gastroenterology 2003;125:815-824. doi: 10.1016/s0016-5085(03)01054-0.

[78] Chavarria L, Oria M, Romero-Gimenez J, Alonso J, Lope-Piedrafita S, Cordoba J. Diffusion tensor imaging supports the cytotoxic origin of brain edema in a rat model of acute liver failure. Gastroenterology 2010;138: 1566-1573. doi: 10.1053/j.gastro.2009.10.003.

[79] Rackayova V, Braissant O, McLin VA, Berset C, Lanz B, Cudalbu C. ${ }^{1} \mathrm{H}$ and ${ }^{31} \mathrm{P}$ magnetic resonance spectroscopy in a rat model of chronic hepatic encephalopathy: in vivo longitudinal measurements of brain energy metabolism. Metab Brain Dis 2016;31:1303-1314. doi: 10.1007/s11011-015-9715-8.

[80] Kabba JA, Xu Y, Christian H, Ruan W, Chenai K, Xiang Y, et al. Microglia: Housekeeper of the central nervous system. Cell Mol Neurobiol 2018;38: 53-71. doi: 10.1007/s10571-017-0504-2.

[81] Garden GA, Möller T. Microglia biology in health and disease. J Neuroimmune Pharmacol 2006;1:127-137. doi: 10.1007/s11481-006-9015-5.

[82] Zemtsova I, Görg B, Keitel V, Bidmon HJ, Schrör K, Häussinger D. Microglia activation in hepatic encephalopathy in rats and humans. Hepatology 2011 ; 54:204-215. doi: 10.1002/hep.24326.

[83] D'Mello C, Le T, Swain MG. Cerebral microglia recruit monocytes into the brain in response to tumor necrosis factoralpha signaling during peripheral organ inflammation. J Neurosci 2009;29:2089-2102. doi: 10.1523/JNEUROSCI.3567-08.2009.
[84] Rodrigo R, Cauli O, Gomez-Pinedo U, Agusti A, Hernandez-Rabaza V, Garcia-Verdugo JM, et al. Hyperammonemia induces neuroinflammation that contributes to cognitive impairment in rats with hepatic encephalopathy. Gastroenterology 2010;139:675-684. doi: 10.1053/j.gastro.2010. 03.040.

[85] Dennis CV, Sheahan PJ, Graeber MB, Sheedy DL, Kril JJ, Sutherland GT. Microglial proliferation in the brain of chronic alcoholics with hepatic encephalopathy. Metab Brain Dis 2014;29:1027-1039. doi: 10.1007/s11011013-9469-0.

[86] Cui Y, Takashima T, Takashima-Hirano M, Wada Y, Shukuri M, Tamura Y, et al. 11C-PK11195 PET for the in vivo evaluation of neuroinflammation in the rat brain after cortical spreading depression. J Nucl Med 2009;50:1904-1911. doi: 10.2967/jnumed.109.066498.

[87] Luo S, Kong X, Wu JR, Wang CY, Tian Y, Zheng G, et al. Neuroinflammation in acute hepatic encephalopathy rats: imaging and therapeutic effectiveness evaluation using ${ }^{11} \mathrm{C}$-PK11195 and ${ }^{18} \mathrm{~F}-\mathrm{DPA}-714$ micro-positron emission tomography. Metab Brain Dis 2018;33:1733-1742. doi: 10.1007/s11011018-0282-7.

[88] Panickar KS, Jayakumar AR, Rama Rao KV, Norenberg MD. Downregulation of the 18-kDa translocator protein: effects on the ammonia-induced mitochondrial permeability transition and cell swelling in cultured astrocytes. Glia 2007;55:1720-1727. doi: 10.1002/glia.20584.

[89] Görg B, Bidmon HJ, Häussinger D. Gene expression profiling in the cerebral cortex of patients with cirrhosis with and without hepatic encephalopathy. Hepatology 2013;57:2436-2347. doi: 10.1002/hep.26265.

[90] Rao KV, Brahmbhatt M, Norenberg MD. Microglia contribute to ammoniainduced astrocyte swelling in culture. Metab Brain Dis 2013;28:139-143. doi: 10.1007/s11011-012-9339-1.

[91] Rangroo Thrane V, Thrane AS, Chang J, Alleluia V, Nagelhus EA, Nedergaard M. Real-time analysis of microglial activation and motility in hepatic and hyperammonemic encephalopathy. Neuroscience 2012;220:247-255. doi: 10.1016/j.neuroscience.2012.06.022.

[92] Balzano T, Dadsetan S, Forteza J, Cabrera-Pastor A, Taoro-Gonzalez L, Malaguarnera $\mathrm{M}$, et al. Chronic hyperammonemia induces peripheral inflammation that leads to cognitive impairment in rats: reversal by antitnfa treatment. J Hepatol 2019. doi: 10.1016/j.jhep.2019.01.008.

[93] Karababa A, Groos-Sahr K, Albrecht U, Keitel V, Shafigullina A, Görg B, et al. Ammonia attenuates LPS-induced upregulation of pro-inflammatory cytokine mrna in co-cultured astrocytes and microglia. Neurochem Res 2017; 42:737-749. doi: 10.1007/s11064-016-2060-4.

[94] Wright G, Swain M, Annane D, Saliba F, Samuel D, Arroyo V, et al. Neuroinflammation in liver disease: sessional talks from ISHEN. Metab Brain Dis 2016;31:1339-1354. doi: 10.1007/s11011-016-9918-7.

[95] Merino ], Aller MA, Rubio S, Arias N, Nava MP, Loscertales M, et al. Gut-brain chemokine changes in portal hypertensive rats. Dig Dis Sci 2011;56:23092317. doi: 10.1007/s10620-011-1625-y.

[96] McMillin M, Frampton G, Thompson M, Galindo C, Standeford H, Whittington $E_{\text {, }}$ et al. Neuronal CCL2 is upregulated during hepatic encephalopathy and contributes to microglia activation and neurological decline. J Neuroinflammation 2014;11:121. doi: 10.1186/1742-2094-11-121.

[97] Zhang L, Tan J, Jiang X, Qian W, Yang T, Sun X, et al. Neuron-derived CCL2 contributes to microglia activation and neurological decline in hepatic encephalopathy. Biol Res 2017;50:26. doi: 10.1186/s40659-017-0130-y.

[98] McMillin M, Grant S, Frampton G, Andry S, Brown A, DeMorrow S. Fractalkine suppression during hepatic encephalopathy promotes neuroinflammation in mice. J Neuroinflammation 2016;13:198. doi: 10.1186/s12974-0160674-8.

[99] Shawcross DL, Davies NA, Williams R, Jalan R. Systemic inflammatory response exacerbates the neuropsychological effects of induced hyperammonemia in cirrhosis. J Hepatol 2004;40:247-254. doi: 10.1016/j.jhep. 2003.10.016.

[100] Butterworth RF. The liver-brain axis in liver failure: neuroinflammation and encephalopathy. Nat Rev Gastroenterol Hepatol 2013;10:522-528. doi: 10.1038/nrgastro.2013.99.

[101] Lambertsen $\mathrm{KL}$, Biber $\mathrm{K}$, Finsen $\mathrm{B}$. Inflammatory cytokines in experimental and human stroke. J Cereb Blood Flow Metab 2012;32:1677-1698. doi: 10. 1038/jcbfm.2012.88.

[102] Rubio-Perez JM, Morillas-Ruiz JM. A review: inflammatory process in Alzheimer's disease, role of cytokines. ScientificWorldJournal 2012;2012: 756357. doi: 10.1100/2012/756357.

[103] Odeh M. Pathogenesis of hepatic encephalopathy: the tumour necrosis factor-alpha theory. Eur J Clin Invest 2007;37:291-304. doi: 10.1111/j. 1365-2362.2007.01778.x.

[104] Jiang W, Desjardins P, Butterworth RF. Cerebral inflammation contributes to encephalopathy and brain edema in acute liver failure: protective effect of minocycline. J Neurochem 2009;109:485-493. doi: 10.1111/j.1471-4159. 2009.05981.x.

[105] Bernal W, Donaldson P, Underhill J, Wendon J, Williams R. Tumor necrosis factor genomic polymorphism and outcome of acetaminophen 


\section{Jaeger V. et al: Astrocytes and microglia in encephalopathy}

(paracetamol)-induced acute liver failure. J Hepatol 1998;29:53-59. doi: 10.1016/s0168-8278(98)80178-5.

[106] Chastre A, Bélanger M, Beauchesne E, Nguyen BN, Desjardins $P_{r}$ Butterworth RF. Inflammatory cascades driven by tumor necrosis factoralpha play a major role in the progression of acute liver failure and its neurological complications. PLoS One 2012;7:e49670. doi: 10. 1371/journal.pone.0049670.

[107] Agusti A, Hernández-Rabaza V, Balzano T, Taoro-Gonzalez L, Ibañez-Grau A, Cabrera-Pastor $A$, et al. Sildenafil reduces neuroinflammation in cerebellum, restores GABAergic tone, and improves motor in-coordination in rats with hepatic encephalopathy. CNS Neurosci Ther 2017;23:386-394. doi: 10 . 1111/cns.12688.

[108] McMillin M, Grant S, Frampton G, Petrescu AD, Williams E, Jefferson B, et al. Elevated circulating TGF $\beta 1$ during acute liver failure activates TGF $\beta$ R2 on cortical neurons and exacerbates neuroinflammation and hepatic encephalopathy in mice. J Neuroinflammation 2019;16:69. doi: 10.1186/s12974019-1455-y.

[109] Malaguarnera M, Llansola M, Balzano T, Gómez-Giménez B, Antúnez-Muñoz C, Martínez-Alarcón $\mathrm{N}$, et al. Bicuculline reduces neuroinflammation in hippocampus and improves spatial learning and anxiety in hyperammonemic rats. Role of glutamate receptors. Front Pharmacol 2019;10:132. doi: 10 . 3389/fphar.2019.00132
[110] Chavarria L, Cordoba J. Encephalopathy and liver transplantation. Metab Brain Dis 2013;28:285-292. doi: 10.1007/s11011-012-9350-6.

[111] Garcia-Martinez R, Rovira A, Alonso ], Jacas C, Simón-Talero M, Chavarria L, et al. Hepatic encephalopathy is associated with posttransplant cognitive function and brain volume. Liver Transpl 2011;17:38-46. doi: 10.1002/It. 22197.

[112] Gerber T, Schomerus H. Hepatic encephalopathy in liver cirrhosis: pathogenesis, diagnosis and management. Drugs 2000;60:1353-1370. doi: 10. 2165/00003495-200060060-00008.

[113] Patidar KR, Bajaj JS. Antibiotics for the treatment of hepatic encephalopathy. Metab Brain Dis 2013;28:307-312. doi: 10.1007/s11011-013-9383-5.

[114] Zacharias HD, Zacharias AP, Gluud LL, Morgan MY. Pharmacotherapies that specifically target ammonia for the prevention and treatment of hepatic encephalopathy in adults with cirrhosis. Cochrane Database Syst Rev 2019;6:CD012334. doi: 10.1002/14651858.CD012334.pub2.

[115] Wijarnpreecha $K$, Werlang $M$, Panjawatanan P, Kroner PT, Cheungpasitporn $\mathrm{W}$, Lukens FJ, et al. Association between sarcopenia and hepatic encephalopathy: A systematic review and meta-analysis. Ann Hepatol 2019. doi: 10. 1016/j.aohep.2019.06.007.

[116] Piotrowski D, Boroń-Kaczmarska A. Bacterial infections and hepatic encephalopathy in liver cirrhosis-prophylaxis and treatment. Adv Med Sci 2017; 62:345-356. doi: 10.1016/j.advms.2016.11.009. 